15

\title{
Iron and coronary heart disease
}

\author{
Iron's role is undecided $/$
}

Several strands of evidence suggest a role for iron in heart disease. For example, the massive accumulation of iron that occurs in genetic haemochromatosis and thalassaemia is a well recognised cause of cardiomyopathy. Furthermore, it has been suggested that iron may be associated with coronary heart disease and that this may occur at quite modest concentrations of stored iron. ${ }^{1-4}$

In 1981 Sullivan hypothesised that the difference in the rates of heart disease between men and women could be explained by their different concentrations of stored iron. ${ }^{1}$ Premenopausal women in affluent societies enjoy relative protection from heart disease compared with men, although this relative protection ends with the menopause. Iron stores, as assessed by the serum ferritin concentration, rise steadily in men after adolescence, but in women they remain low before rising after the age of $45 .{ }^{5}$ These changes parallel the incidence of coronary heart disease in the West. ${ }^{2}$ Additionally, Sullivan postulated that the low rates of heart disease in developing countries may be related to the high prevalence of iron deficiency in these populations. ${ }^{1}$ This hypothesis was supported by Lauffer, who reported a correlation $(r=0.55$, $\mathrm{p}<0.025)$ between the median value of hepatic stored iron and the mortality from ischaemic heart disease in different countries. ${ }^{3} \mathrm{He}$ concluded that it may be possible to identify people at risk of coronary heart disease and reduce this risk by venesection. The low iron stores in some populations may, however, be due to a poor diet: such diets may be less atherogenic because of factors other than iron content.

The debate has intensified after the recent report by Salonen et al that modestly raised serum ferritin concentrations increased the risk of acute myocardial infarction. ${ }^{4}$ In a prospective study of eastern Finnish men, those with a serum ferritin concentration above $200 \mu \mathrm{g} / \mathrm{l}$ were more than twice as likely to have an acute myocardial infarction as men with a serum ferritin concentration below this figure. The risk was highest in men who had both a serum ferritin concentration above $200 \mu \mathrm{g} / \mathrm{l}$ and a serum low density lipoprotein cholesterol concentration above $5.0 \mathrm{mmol} / \mathrm{l}$.

Increased serum ferritin concentrations reflect increased iron stores but may also be related to inflammatory responses and alcohol consumption. ${ }^{5}$ These variables did not, however, explain the differences in ferritin concentrations or the rates of myocardial infarction. ${ }^{4}$ Dietary iron intake was also significantly associated with the risk of disease.
The proposed mechanism implicating iron in the pathogenesis of coronary heart disease includes post-secretory modifications of low density lipoprotein that increase its atherogenic potential. ${ }^{6}$ Oxidative modification of low density lipoprotein depends on the concentrations of iron and copper and can be inhibited in vitro by metal chelators. Modified low density lipoprotein is immunogenic, and antibodies are detectable in serum and can recognise material in atherosclerotic but not in normal arteries. ${ }^{6} \mathrm{~A}$ case-control study has shown that the titre of these autoantibodies independently predicts the progression of carotid atherosclerosis, ${ }^{7}$ but similar results have not been reported for coronary heart disease. To shed light on whether modification of lipoprotein is causally related to atherosclerosis (rather than being an epiphenomenon) the role of antioxidants has been studied.

Probucol, a lipid lowering agent with antioxidant properties, inhibits the development of atherosclerosis in hypercholesterolaemic rabbits independently of its cholesterol lowering effect. ${ }^{8}$ Trials in humans are awaited, but reports of an inverse relation between angina and concentrations of naturally occurring antioxidants, such as vitamins $\mathrm{C}$ and $\mathrm{E}$, are of interest. ${ }^{9}$ Even if modified lipoproteins are shown to have an important role in atherogenesis, the importance of iron is still unclear. Although iron may play a part in the modification of lipoprotein, this does not necessarily imply that iron induces or drives the process, and the availability of the iron at the sites of peroxidation is unclear.

Experimental evidence also implicates iron in the genesis of the myocardial ischaemic or reperfusion injury. Iron may have a role in the production of free radicals that mediate tissue injury. ${ }^{10}$ Treatment with desferrioxamine can reduce the degree of myocardial injury, ${ }^{11}$ and, conversely iron loading increases the susceptibility of the rat heart to reperfusion damage.

If iron has a substantial role in the pathogenesis of atherosclerosis and ischaemic heart disease, subjects homozygous or heterozygous for haemochromatosis might be expected to experience high rates of coronary heart disease. But evidence of this is lacking. ${ }^{12}{ }^{13}$ The role of iron in the pathogenesis of coronary heart disease therefore remains undecided.

This raises questions about optimal body iron stores and optimal dietary requirements, but, until we know more about control of the availability of iron in tissues and the toxicity 
of iron, no firm conclusions can be drawn. Until they can be, it seems prudent to prescribe iron only for iron deficiency and during pregnancy.

Queensland Institute of Medical Research,

Brisbane,

Queensland 4029, Australia

1 Sullivan JL. Iron and the sex difference in heart disease risk. Lancet 1981;i:1293-4.

2 Sullivan IL. The iron paradigm of ischemic heart disease. Am Hean $f$ 1989;117:1177-88.

3 Lauffer RB. Iron stores and the international variation in mortality from coronary artery disease. Med Hypotheses 1990;35:96-102.

4 Salonen JT, Nyyssőnen K, Korpela H, Tuomilehto J, Seppănen R, Salonen R. High stored iron levels are associated with excess risk of myocardial infarction in eastern Finnish men. Circulation 1992;86:803-11
5 Leggett BA, Brown NN, Bryant SJ, Duplock L, Powell LW, Halliday JW. Factors affecting the concentrations of ferritin in serum in a healthy Australian population. Clin Chem 1990;36: $1350-5$

6 Steinberg D, Parthasarathy S, Carew TE, Khoo JC, Witztum JL. Beyond cholesterol. Modifications of low-density lipoprotein that increase its atherogenicity. $N$ Engl f Med 1989;320:915-24.

7 Salonen JT, Ylä-Herttuala S, Yamamoto R, Butler S, Korpela H, Salonen R, et al. Autoantibody against oxidised LDL and progression of carotid atherosclerosis. Lancet 1992;339:883-7.

8 Carew TE, Schwenke DC, Steinberg D. Antiatherogenic effect of probucol unrelated to its hypocholesterolemic effect: evidence that antioxidants in vivo can selectively inhibit low density lipoprotein degradation in macrophage-rich fatty streaks and slow the progression of atherosclerosis in the Watanabe heritable hyperlipidemic rabbit. Proc Natl Acad Sci USA 1987;84: 7725-9.

9 Riemersma RA, Wood DA, MacIntyre CCA, Elton RA, Gey KF, Oliver MF. Risk of angin pectoris and plasma concentrations of vitamin A, C, and E and carotene. Lancet 1991;337:1-5.

10 Halliwell B. Current status review: free radicals, reactive oxygen species and human disease: a critical evaluation with special reference to atherosclerosis. British foumal of Experimental Pathology 1989;70:737-57.

11 Williams RE, Zweier JL, Flaherty JT. Treatment with deferoxamine during ischemia improves functional and metabolic recovery and reduces reperfusion-induced oxygen radical generation in rabbit hearts. Circulation 1991;83:1006-14.

12 Bradbear RA, Bain C, Siskind V, Schofield FD, Webb S, Axelsen E, et al. Cohort study of internal malignancy in genetic hemochromatosis and other chronic nonalcoholic liver diseases. $\mathcal{f} \mathrm{Nat}$ Cancer Inst 1985;75:81-4.

13 Powell LW, Halliday JW, Wilson A, Siskind V, Bain C, Bradbear RA. Proceedings of the 4th international conference on haemochromatosis and clinical problems in iron metabolism. Israel, 1993.

\section{Should purchasers pay for psychotherapy?}

\section{All therapies must prove their worth; some have found this easier than others}

Psychotherapy is a nebulous term referring to a seemingly infinite variety of "talking cures" of differing intensity, duration, and theoretical sophistication. The new rigours of the purchaser-provider split mean that all such treatments will now be subject to the same cost-benefit analyses that have restricted the range of psychotherapy funded by private health insurance. Psychotherapies that controlled trials have shown to be effective and safe and to offer good value for money will be in a stronger position to argue their case in the health marketplace. But among psychotherapists attitudes to cost-benefit analysis are polarised, and information on the utility of the interventions is uneven in quality.

The psychotherapy that has adopted the market approach par excellence is behaviour therapy. The practice of graded exposure to phobic stimuli incorporates the routine measurement of symptoms during treatment and the recording of hours of therapy. Cost-benefit information is thus readily available. Behaviour therapy has proved itself an effective and economical method of treating phobic, obsessive compulsive, sexual, and habit disorders, ${ }^{1}$ providing lasting reductions in symptoms together with measurable economic benefits to patients, families, and the health service. ${ }^{2}$ Employing trained nurse therapists could further reduce its costs. ${ }^{3}$

Cognitive behavioural therapy combines some of the principles of behaviour therapy with a systematic analysis and challenging of the thoughts and beliefs that underlie mood disturbance, low self esteem, or dysfunctional behaviour. This therapy has also been subject to rigorous comparisons with other forms of brief psychotherapy and pharmacotherapy. The results suggest that it is effective for mild or moderate depression, ${ }^{4}$ and may be as effective as, if not better than, antidepressant drugs in preventing relapse. ${ }^{56}$ It has also been used successfully in various neurotic disorders, ${ }^{7}$ such as bulimia nervosa. ${ }^{8}$ Treatment can be given by psychiatrists, psychologists, or nurses (usually supervised by a senior psychologist or psychiatrist).

The role of the dynamic psychotherapies within the new NHS presents the most difficulty for purchasers and managers. The methodological difficulties in assessing the effectiveness of intensive long term analytic therapies, such as defining the nature of treatment and devising control treatments, means that there is a dearth of controlled studies.
Some therapists argue that the aim of this type of therapy is to promote change that is not amenable to simple measurement. These arguments are unlikely to impress purchasers or managers, but psychodynamic psychotherapists in the NHS are finally beginning to provide more convincing arguments. ${ }^{9}$

Although patients improve over the course of long term psychotherapy, ${ }^{10}$ Andrews has noted that, without controlled trials, it is impossible to decide how much improvement is due to the specific effects of treatment and how much is due to a placebo effect or the passage of time. ${ }^{11}$ Andrews also draws attention to the considerable expense, and occasional adverse effects, of long term psychotherapy. An Australian survey found that therapists spent an average of 330 hours with their patients, although they failed to define the indications for such therapy. ${ }^{12}$ The length of therapy, and the shortage of therapists, meant that only a small fraction of those with mental health problems could be treated. Purchasers will justifiably be wary of funding such an inequitable service.

Dynamic psychotherapists may have to consider new methods of working, and new client groups, if they are to meet the challenge of the market. One rarely explored avenue lies in the health of the NHS workforce-an explicit target of The Health of the Nation. NHS psychotherapists must also move beyond the traditional clients of their colleagues in private practice. They may have a role in the management of personality disorders, victims of childhood abuse, and those with abnormal illness behaviour. They must take note that the selection of relatively homogeneous diagnostic groups for studies of the brief psychotherapies has been an important step in assessing efficacy and defining clinical indications in the public health care system. Without a clear target group, and without evidence of efficacy, the long term therapies are vulnerable when it comes to decisions about resources.

Several brief psychodynamic therapies have been developed, which address some of these criticisms. A metaanalysis of 11 studies that used treatment manuals or similar ways of ensuring adherence to treatment concluded that treated patients did significantly better than controls taken from the waiting list, ${ }^{13}$ but the effects of brief psychodynamic therapies differed little from those of placebo, self-help, and 